\title{
Quelle définition des « petits métiers » de la pêche?
}

De l'analyse juridique à l'ethnoécologie des pêcheurs lagunaires languedociens

Which definition for "small trade" fishing? From the legal analysis to an ethnoecology of lagoon fishermen in Languedoc

Nastassia Reyes, Serge Bahuchet et Jean-Dominique Wahiche

\section{CpenEdition Journals}

Édition électronique

URL : https://journals.openedition.org/ethnoecologie/2221

DOI : 10.4000/ethnoecologie.2221

ISSN : 2267-2419

Éditeur

Laboratoire Éco-anthropologie

Référence électronique

Nastassia Reyes, Serge Bahuchet et Jean-Dominique Wahiche, «Quelle définition des «petits métiers » de la pêche ? », Revue d'ethnoécologie [En ligne], 7 | 2015, mis en ligne le 30 juin 2015 consulté le 15 février 2023. URL : http://journals.openedition.org/ethnoecologie/2221 ; DOI : https:// doi.org/10.4000/ethnoecologie.2221

Ce document a été généré automatiquement le 29 septembre 2020.

\section{cc) (1)}

Creative Commons - Attribution - Pas d'Utilisation Commerciale - Pas de Modification 4.0 International - CC BY-NC-ND 4.0

https://creativecommons.org/licenses/by-nc-nd/4.0/ 


\title{
Quelle définition des " petits métiers » de la pêche?
}

\author{
De l'analyse juridique à l'ethnoécologie des pêcheurs lagunaires \\ languedociens \\ Which definition for "small trade" fishing? From the legal analysis to an \\ ethnoecology of lagoon fishermen in Languedoc
}

\author{
Nastassia Reyes, Serge Bahuchet et Jean-Dominique Wahiche
}

Au sortir de la seconde guerre mondiale s'est engagée une exploitation intensive de la ressource halieutique s'appuyant sur l'idée qu'elle était intarissable (Cury \& Miserey, 2008). Témoin de cette période cruciale pour la transformation des métiers de la pêche, Auguste Dupouy discute de l'opposition alors fréquente entre "pêche artisanale » et " pêche moderne », qu'il préconise aussi d'appeler : "pêche industrielle». Selon l'auteur, « est réputée industrielle la pêche pratiquée par un bateau d'au moins 50 tonneaux de jauge brute: au-dessous de ce minimum elle reste artisanale.» (1955: 101). Il précise cependant que ce qui distingue les deux est la prise en charge médicale du pêcheur en cas de blessure à bord. Il ajoute que l'artisanat et l'industrie se distinguent surtout par la manière dont le poisson est transformé et conditionné après capture, plutôt que par la technique de pêche elle-même : «Si l'expression pêche industrielle a un sens, elle ne peut que désigner corrélativement deux choses: une transformation de l'outillage et une régularisation du rendement » $(1955: 103)$. En effet on assiste alors au développement de la technique du chalut par l'accroissement de la propulsion mécanique. L'intensification de la pêche qui en résulte n'est pas sans inquiéter A. Dupouy, qui se demande : cette pêche moderne, disons industrielle, assure un ravitaillement copieux, quelquefois même surabondant, mais à quel prix et pour combien de temps ?» $(1955: 117)$.

2 Ainsi, l'importance économique des gros armements de pêche a longtemps placé cette activité au premier plan. Cependant, dans les années 1990, ce mode d'exploitation montre ses limites en termes de rentabilité économique et d'impact sur les ressources. Depuis, l'attention tend à s'orienter vers les pêches artisanales que les politiques, les écologistes 
et les scientifiques jugent plus respectueuses de l'environnement, donc plus perdurables et socialement plus équitables - sans ignorer toutefois le fait que les small-scale fisheries sont désavantagées car les flottes de la pêche industrielle sont hautement subventionnées et obtiennent un meilleur accès au marché (Jacquet \& Pauly 2008).

Pour ce qui est de la Méditerranée française et notamment le Languedoc-Roussillon, zone d'étude sur laquelle nous nous sommes concentrés, la flotte est composée à $80 \%^{1}$ d'embarcations de petite taille mesurant moins de 12 mètres de long (Lecou 2010). Ces embarcations sont appelées localement les «petits métiers ». Cependant, en dépit de leur importance, on relève une réelle imprécision dans la définition des catégories professionnelles pratiquant cette activité halieutique.

Selon l'acception la plus répandue, « la pêche aux petits métiers » désigne l'ensemble des navires de pêche, à l'exception des chalutiers qui détiennent une licence de chalutage et des thoniers-sardiniers qui détiennent une licence de pêche aux espèces pélagiques (Guillou et al. 2002 : 4). Il s'agit donc d'une catégorie qui ne trouve une existence que par contraste avec les deux autres activités. De plus, cette définition ne révèle rien quant à son contenu. On constate également que, dans les divers textes officiels, plusieurs termes sont présents pour parler de ces petites pêches méditerranéennes. Cependant, on remarque un manque de concordance entre la nomenclature des lois françaises et celle des textes européens.

Dans ce contexte, l'objectif de cet article est de pousser l'analyse pour parvenir à comprendre, à partir d'une approche en anthropologie juridique et en ethnoécologie, ce que recouvre cette catégorie des pêches aux "petits métiers». Méthodologiquement, notre démarche est inductive. Nous exposerons la terminologie présente dans les textes juridiques européens et français pour parler des pêches aux " petits métiers ». Puis, afin de mener une analyse fine de ce à quoi correspond ce segment, nous avons choisi de nous concentrer plus particulièrement sur les activités de pêche dans les lagunes languedociennes, en détaillant les techniques. En effet, les techniques de pêche sont les moyens par lesquels le pêcheur accède à la ressource halieutique (poissons, mollusques et crustacés) et se l'approprie (Geistdoerfer 1974). Elles sont le produit de l'homme qui a su les adopter ou les élaborer d'après les observations qu'il a faites sur le milieu et sur les comportements des espèces recherchées (Geistdoerfer 1977, 1985 ; Bahuchet 1992). Nous chercherons ici à dépasser la seule dimension technique pour donner une place à l'humain en présentant la manière dont le pêcheur considère l'exercice de chacune de ces techniques. Pour finir, nous développerons le lien étroit qu'entretiennent les pêcheurs aux « petits métiers » avec le milieu qu'ils exploitent. La description des techniques nous permet de comprendre avec précision le caractère des interactions entre l'homme et la nature. À partir de ce travail descriptif, nous pourrons proposer une définition heuristique des « petits métiers » de la pêche qui corresponde aux réalités du terrain.

\section{Une question de définition : comment parle-t-on des « petits métiers » dans les textes juridiques européens et français?}

6 Dans le dernier texte européen de la Politique commune des pêches $(\mathrm{PCP})^{2}$ les expressions "pêche de petite échelle ", "pêche artisanale " et "pêche côtière " sont utilisées simultanément sans que leurs sens ne se superposent vraiment. 
7 L'expression "pêche de petite échelle » suggère un échelonnement alors qu'aucun gradient de référence ne permet de déterminer ce qui relève du « petit » ou du « grand ». Cette séparation est d'ailleurs qualifiée d'«arbitraire» dans un rapport de l'Union européenne (Macfadyen et al. 2011). Elle s'avère également peu précise dans la mesure où elle offre une variété d'interprétation quant à savoir si elle se réfère à des aspects techniques, tels que la taille du navire, ou à des aspects économiques, tels que les quantités débarquées, ou bien à toute autre considération.

On trouve également l'expression "pêche artisanale ", en partie définie précédemment. C'est l'expression la plus communément utilisée dans le contexte actuel de diminution de certaines ressources marines. En effet, le simple fait d'associer à n'importe quel terme celui d'artisanal contribue à évoquer un imaginaire correspondant à un savoir-faire particulier, lié à un travail soigneux, méticuleux et même " authentique », fondé sur une organisation familiale de petite production. Or, les données manquent au sujet de ces pêcheries et le risque d'idéalisation est important. Mais l'effort de clarification de cette expression au sein de la politique européenne des pêches est encore loin d'arriver à son terme. En effet, en raison des différentes interprétations que donnent les États membres de ce qui constitue une pêche artisanale en fonction des contextes nationaux, l'Union européenne a une réelle difficulté à dégager une définition. Ainsi, alors même que la PCP reconnaît l'importance socio-économique de cette pêcherie, on constate qu'aucune définition n'est fournie. Par ailleurs, au niveau national, la " pêche artisanale » est définie autour du statut social et fiscal du patron embarqué ${ }^{3}$ et correspond aux navires de pêches d'une longueur inférieure à 25 mètres $^{4}$. Dans ce contexte, la technique de pêche au chalut se voit incluse au sein de ce segment. Or, il s'agit d'un art de pêche dit traînant que les pêcheurs aux « petits métiers » considèrent être un métier de pêche résolument différent de celui qu'ils pratiquent. Ainsi, cette expression sujette à des interprétations multiples revêt-elle un sens très général qui est imprégné de représentations sociales ambiguës.

9 Enfin, on trouve également l'expression "pêche côtière " qui est la seule à être communément répertoriée dans les quatre textes successifs de la Politique commune des pêches ${ }^{5}$. À la différence des précédentes, l'expression « pêche côtière » met l'accent sur la dimension spatiale de la proximité avec la côte. Au niveau européen, cette expression comprend une dimension juridique forte dans la mesure où elle fait référence aux navires ayant accès aux eaux relevant de la souveraineté et de la juridiction des États côtiers c'est-à-dire, situées en deçà d'une limite maximale de 12 milles marins ${ }^{6}$. Cependant, rapportée à l'échelle de la Méditerranée française cette définition n'est pas adaptée. En effet, certains navires exerçant la pêche aux « petits métiers » peuvent s'éloigner jusqu'à 20 milles marins de la terre la plus proche ${ }^{7}$.

10 En complément de cette expression on trouve dans quelques autres règlements européens ${ }^{8}$ l'expression « petite pêche côtière ». Celle-ci est la seule définition réellement établie. Elle désigne la pêche pratiquée par des navires dont la longueur hors tout est inférieure à 12 mètres et qui n'utilisent pas d'engins remorqués ${ }^{9}$. Bien que partielle, cette définition semble être celle qui, à l'échelle européenne, correspond le plus à l'activité des « petits métiers » que l'on rencontre en Méditerranée française.

11 Dans les textes juridiques nationaux on trouve les expressions de "petite pêche » et de "pêche côtière » qui semblent s'approcher de l'expression européenne par l'emploi des mêmes termes. On pourrait donc croire à une concordance des significations. Cependant, même si elles sont proches de l'expression européenne, elles s'avèrent en fait s'employer dans le domaine spécifique de la navigation maritime. En fait, l'expression «petite 
pêche " correspond alors aux navires qui effectuent des sorties en mer d'une durée inférieure ou égale à 24 heures, tandis que " pêche côtière » désigne les navires effectuant des sorties en mer comprises entre 24 et 96 heures $^{10}$. Or, les pêcheurs aux «petits métiers » effectuent des sorties à la journée et cherchent à valoriser cette caractéristique en insistant sur la fraîcheur des poissons qu'ils débarquent. Bien que ces expressions soient proches de l'expression employée dans le texte européen précédemment cité, on constate que le sens qui leur est attribué n'est pas le même. C'est étonnamment dans un autre domaine que l'on retrouve au niveau national une définition qui fait écho à celle donnée par l'Union européenne. En effet, le décret 2011-697 du 20 juin 2011 du Code général des impôts définit la notion de "petite pêche côtière " comme étant « la pêche pratiquée au moyen de bateaux dont la longueur hors tout est inférieure à douze mètres, n'utilisant que des engins non remorqués et dont la sortie en mer n'excède pas 24 heures. " Cette définition mobilise ainsi trois critères essentiels (taille du navire, type d'engin de pêche employé et durée de sortie en mer) mais non suffisants, qui caractérisent la pêche aux « petits métiers » de Méditerranée française.

Finalement, au lieu d'être une simple expression utilisée localement, l'expression « petits métiers » trouve une existence juridique dans l'arrêté du 19 décembre 1994 portant réglementation technique pour la pêche professionnelle en Méditerranée continentale. L'article 1 définit les "petits métiers côtiers polyvalents " comme tout mode de pêche, autre que la pêche au chalut, à la senne, au gangui et à la drague, pratiqué à partir de navires d'une longueur hors tout inférieure ou égale à 18 mètres. Cet article est complété par un second qui désigne par " petits métiers du large polyvalents » tout mode de pêche, autre que la pêche au chalut, à la senne, au gangui et à la drague, pratiqué à partir de navires d'une longueur hors tout supérieure à 18 mètres et inférieure à 25 mètres. Cette définition a l'avantage d'insister sur la principale caractéristique de ce segment: la polyvalence. Elle reprend également deux autres critères : en excluant les arts traînants et en se référant à une certaine dimension du navire. Néanmoins, elle reste trop large et ne convient pas tout à fait à la réalité du terrain telle que la conçoivent les pêcheurs aux " petits métiers ». Pour eux, les " petits métiers » ne mesurent en général pas plus de 12 mètres; toutefois ils admettent, sous certaines conditions, que des navires d'une limite maximale de 15 mètres puissent également être inclus au sein de l'activité des "petits métiers".

Nous avons donc pris comme point de référence l'expression locale "petits métiers ", telle qu'employée par les pêcheurs auprès desquels nous travaillons pour désigner l'activité halieutique qu'ils exercent. Du point de vue des pratiques et de la manière de travailler des pêcheurs, elle correspond à un ensemble cohérent. Nous avons donc recherché quelles étaient les expressions utilisées aux niveaux national et communautaire pour évoquer cette activité de pêche. Une multitude d'expressions ont pu être répertoriées et nous avons constaté qu'elles ne concordent pas totalement les unes avec les autres. En effet, tandis que certaines sont présentes indépendamment dans les textes législatifs français et dans les textes législatifs européens, d'autres y figurent simultanément. On constate, de plus, qu'il y a des glissements dans l'utilisation des termes. Après analyse nous pouvons conclure que ces occurrences communes n'induisent pas pour autant que ce soit la même sémantique qui leur est attribuée. Les expressions ont parfois des sens différents dès lors que l'on change d'échelle et de point de référence. Sans qu'elles coïncident vraiment, on remarque que les définitions européennes et nationales de "petite pêche côtière » ont une sémantique qui se rapproche. Néanmoins, 
la définition nationale s'inscrit au sein du Code général des impôts, ce qui l'inscrit dans un domaine particulier un peu en marge de celui mobilisé dans le domaine de la gestion et de l'exercice des pratiques de pêche. Enfin, on constate que l'on ne se réfère pas au même vocabulaire selon qu'il est question de navigation, de taille du navire, des caractéristiques des engins, etc. La terminologie relative à ce segment d'activité n'a, en effet, jamais été codifiée dans son ensemble. Ainsi le sens local attribué à l'expression "petits métiers" semble issu de la juxtaposition ou de la superposition de différents critères et donc de différentes expressions. Pour comprendre les limites de chacune de ces expressions, nous avons choisi de partir de l'analyse des pratiques de pêche locales.

\section{Présentation de l'approche ethnoécologique : les modes d'interactions avec les acteurs}

Les données présentées ici sont issues d'un travail de terrain ${ }^{11}$ qui a commencé en 2012 et dont la durée cumulée s'élève à dix mois. Ces données s'appuient sur une approche ethnoécologique qui se définit comme étant: « l'étude des relations qui lient une société humaine au milieu naturel qui l'entoure [...]; elle doit se baser d'une part sur la perception que peuvent avoir les individus eux-mêmes de ce milieu naturel et de leurs actes, d'autre part sur une analyse extérieure du milieu et des actions des hommes » (Bahuchet 1986).

Le travail d'enquête a commencé par l'identification des lieux en rapport plus ou moins direct avec les activités halieutiques: le quai d'amarrage des navires, les lieux de socialisation des pêcheurs, de stockage des engins et de vente de captures. Cela nous a permis d'acquérir une compréhension de l'espace et des déplacements des pêcheurs. Le réseau de connaissance que nous avons tissé auprès des pêcheurs professionnels s'est construit au fil des rencontres et sans passer par des institutions identifiées. Nous avons préféré opérer de cette manière de façon à conserver une distance vis-à-vis des enjeux et rapports de forces entre institutions.

Sur le terrain, nous avons essayé de nous faire à ce que Malinowski appelait les «banalités de la vie quotidienne » (1963 [1922]). Nous avons suivi et participé, autant que possible, à toutes les activités en rapport avec la pêche (le démaillage des filets, la vente à quai, le dépôt de poissons à la criée, etc.).

17 L'adaptation au rythme de vie des pêcheurs nous a permis d'identifier les moments pertinents pour réaliser les entretiens. Une attention particulière a été portée à ce qu'ils soient menés dans le contexte quotidien des pêcheurs. En exerçant leur activité lors des embarquements en mer ou simplement à terre, les pêcheurs nous ont offert des explications précises sur les techniques, les espèces pêchées et sur les savoirs écologiques qu'ils en ont.

\section{"L'étang » : un ancrage territorial et identitaire fort de l'activité de pêche en Languedoc-Roussillon}

« ...il est en effet bien rare qu'en venant de l'intérieur des terres pour aller vers la mer on ne rencontre pas un étang d'eau saumâtre qui complique l'accès au littoral...» (Giovannoni 1995 : 17) 


\section{Une activité de prélèvement sur un écosystème particulier}

18 La particularité la plus remarquable des côtes du Languedoc est que le rivage se fait hésitant. Derrière le cordon littoral, qui s'étend sur près de 200 kilomètres depuis les Pyrénées jusqu'aux terres de Camargue, se trouve une multitude de lagunes qui longent la façade maritime d'une manière quasi continue. L'ensemble des lagunes du golfe du Lion, nommées localement "étangs", couvre une surface totale d'environ 65000 hectares (Campillo 1992).

19 L'étang se présente comme un milieu original où la faune aquatique est plus variée, et surtout plus dense qu'en mer. En effet, « la richesse faunistique des étangs languedociens est étroitement liée à la façon dont s'organise - à l'échelle du golfe du Lion tout entier les cycles biologiques qui, en interaction entre la mer et les étangs, ordonnent les activités halieutiques » (Giovannoni $1995: 25)$. Ces zones font l'objet d'une activité de pêche particulièrement intense, rythmée par la présence de nombreuses espèces cibles ${ }^{12}$ qui se concentrent à l'intérieur des étangs durant des périodes plus ou moins longues. Elles «ne séjournent dans le milieu lagunaire qu'à certaines époques de leur cycle de vie, qui correspond généralement aux phases trophiques, alors que les phases reproductrices ne se déroulent qu'en mer » (Farrugio \& Le Corre 1985 : 14-15). L'importance écologique des étangs réside dans le fait que ce sont des nurseries pour de nombreuses espèces de poissons ${ }^{13}$. Or, au moment de ces phases de reproduction, les poissons vont se regrouper. Ces moments vont ponctuer l'activité des pêcheurs car il s'agit de périodes propices qui permettent, généralement, de réaliser des quantités de captures favorables. Ces particularités physiques et écologiques des étangs vont alors influer sur l'activité des pêcheurs et donc sur les rapports qu'ils auront avec le milieu.

\section{Un adret pour les populations locales}

Historiquement, l'activité de pêche en Languedoc a commencé par se développer dans les étangs (Féral 1980). Les villages anciens du littoral ne se sont pas construits sur le lido ${ }^{14}$ mais sur les berges, à l'intérieur des étangs. Le rivage intérieur s'est "adossé à un territoire fertile, au pied des riches collines couvertes de vignes et d'oliviers, côtoyé par la voie Domitienne qui, depuis les Romains, était la route obligée d'Italie en Espagne. Il présentait une succession de villes littorales et de ports tels que Mèze-Mesua, BouziguesPosyguim, Frontigan-Forum Domitii, Lattes-Castel-Latara, Aigues-Mortes-Aquae-mortoe, Saint-Gilles-Rhodanusia, Arles-Arelate qui ont joué un rôle maritime de premier ordre jusqu'à ces derniers siècles » (Lenthéric 1876 : 285).

21 Protégés des rigueurs de la mer et des invasions pirates, les étangs ont été des lieux privilégiés pour l'installation des pêcheurs locaux.

Dans la mesure où il est difficile de couvrir par une démarche anthropologique une étendue aussi vaste que l'ensemble de la région Languedoc-Roussillon, nous avons choisi de localiser l'étude sur le quartier de la Pointe Courte à Sète et sur la ville de Palavas-lesFlots. Nous ferons ici un court détour par l'histoire de ces localités pour une meilleure compréhension du développement des activités de pêche qui sont pratiquées aujourd'hui. Le choix s'est tout d'abord porté sur la ville de Sète, en tant que premier port de pêche en Méditerranée française en volume de captures débarquées (FranceAgriMer 2013). C'est un lieu particulier tant par la diversité des pêcheurs et des techniques de pêches qui se 
côtoient (présence de chalutiers, de thoniers-senneurs et de "petits métiers» de la pêche) que par les rapports sociaux et les problématiques qui se dégagent. Dans ce contexte, le quartier de la Pointe Courte est un lieu emblématique de l'activité de pêche développée sur l'étang de Thau. Le quartier s'est constitué à l'arrivée de pêcheurs venus faire fortune lors de la création du port de Sète en 1666 (Giovannoni 1995). À cette époque, de grands travaux se multiplient laissant une espace inhabité au bord du canal. Les pêcheurs commenceront par y construire quelques baraquements afin d'entreposer leur matériel. Ce n'est que plus tard que leur nombre se multipliera et que les baraquements finiront par être utilisés comme habitat principal (Giovannoni 1995). Aujourd'hui ce quartier attire la curiosité des estivants qui viennent visiter ce lieu souvent qualifié de "pittoresque» par de nombreux guides touristiques. Il suffit de pénétrer dans le quartier, de porter son attention sur les façades des maisons et sur le nom des rues pour s'apercevoir qu'il est marqué par l'activité de pêche. On emprunte le quai du mistral. À quelque 50 mètres, on tourne à droite dans la traverse des pêcheurs. On se retrouve alors sur le quai Jean Vallée, célèbre jouteur nautique ${ }^{15}$ de la région. On constate à cet endroit une accumulation d'engins de pêche et de petites barques d'en moyenne 6 mètres.

24 Aujourd'hui encore, certains pêcheurs habitent le quartier de la Pointe Courte mais c'est surtout le cas des pêcheurs retraités. Les professionnels en exercice empruntent pour la majorité d'entre eux leur voiture pour rejoindre leur logement situé à l'extérieur du quartier. Le lieu d'habitation des pêcheurs, autrefois très localisé, est aujourd'hui plus dispersé. Cependant, cela ne remet pas en cause l'attachement des pêcheurs à leur quartier: il reste un lieu de référence de l'activité de pêche et de la transmission générationnelle du métier.

Afin de s'extraire du particularisme de l'organisation de la pêche sétoise, il a été décidé de mener une étude comparative. Le choix s'est porté sur le port de Palavas-les-Flots en raison de l'importance que tient cette activité dans l'histoire locale. Entouré de dix étangs, il est à l'origine un village de pêcheurs. «Les premiers établissements humains remontent à 1811. Un plan cadastral de la commune de Mauguio mentionne, à cette date, 14 cabanes [de pêcheurs] établies sur la rive gauche du Lez » (Rieucau 1990 : 57). Dans le village naissant, concrétisé par la construction de maisons en dur, on dénombre, sur 202 habitants recensés, 166 personnes qui vivent de la pêche (Féral 1980).

Palavas-les-Flots est la «banlieue bleue» de Montpellier (Rieucau 1990 : 53). Proche du littoral, cette ville offre aujourd'hui de nombreuses activités récréatives, nautiques, résidentielles. Autrefois, les familles montpelliéraines s'y rendaient chaque année pour profiter des plages. C'est encore le cas aujourd'hui. Plus récemment, s'ajoute à celles-ci une masse de touristes contribuant à faire de la ville une station balnéaire. Pour la plupart, les pêcheurs y résident à l'année. Autrefois concentré dans le cœur urbain, leur lieu de résidence est aujourd'hui là aussi plus dispersé, tandis que la localisation de leurs bateaux de pêche est souvent fonction de l'étang qu'ils exploitent.

D'un port et d'un étang à l'autre, l'histoire des peuplements humains diffère quelque peu. Cependant, cette mise en contexte permet de montrer que les activités de pêche en lagune s'inscrivent dans un contexte historique et territorial précis. 


\section{Quelques techniques de pêche comme autant de manières différentes de travailler}

Pour pêcher dans les étangs, les pêcheurs utilisent des petites barques à fond plat mesurant environ six mètres. Autrefois exclusivement conduites à la rame, elles sont aujourd'hui munies d'un moteur hors-bord, dont la puissance moyenne est de $30 \mathrm{cv}$. Seul un ou tout au plus deux hommes embarquent à leur bord. Ces embarcations sont dépourvues de matériel électronique et de mécanisation ${ }^{16}$ et les pêcheurs travaillent à la force des bras.

Figure 1 : Bateaux armés à la pêche aux « petits métiers » d'étang, Sète

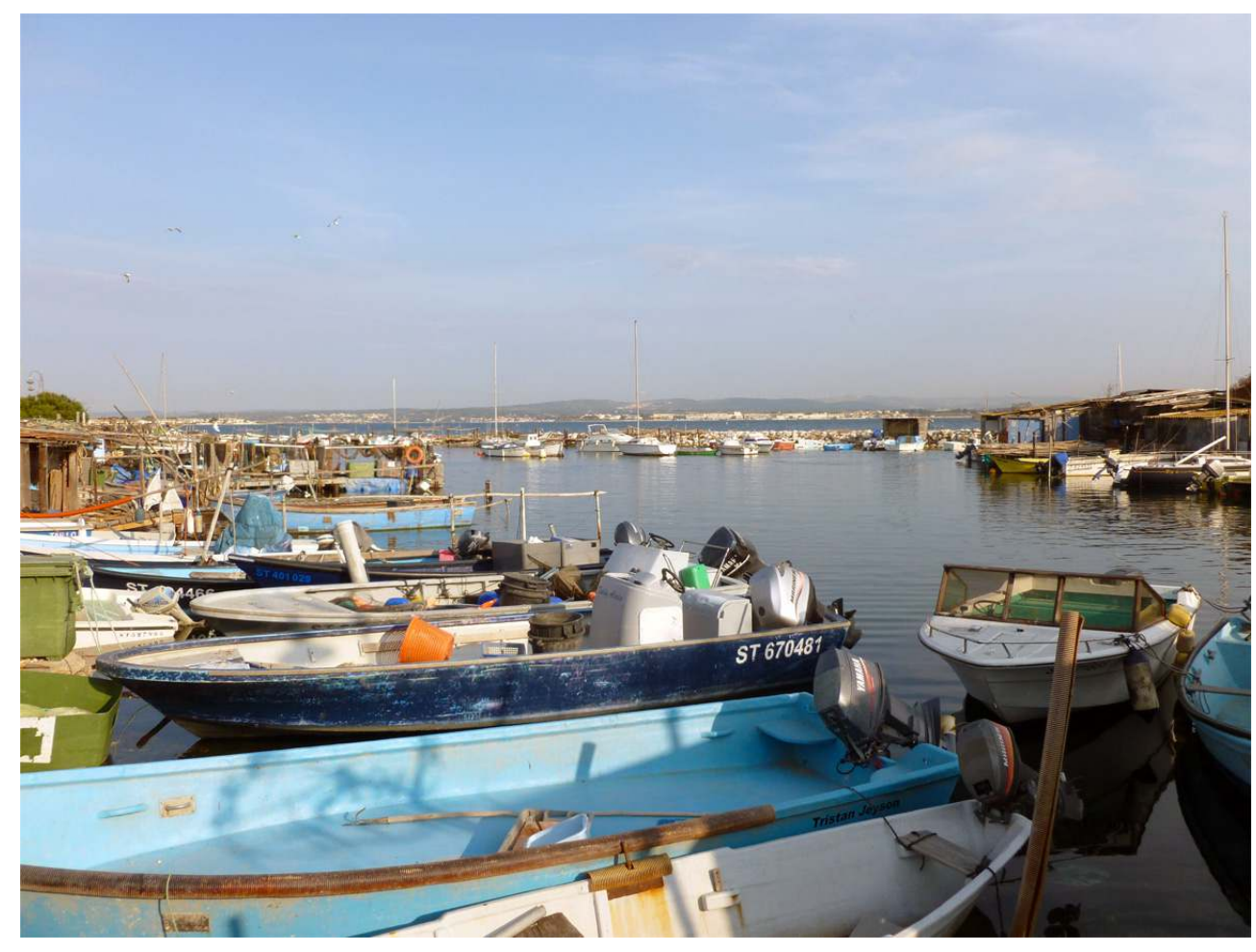

(c) N. Reyes

En lagune, quelques-unes des principales techniques exercées par les pêcheurs aux «petits métiers » ne sont pratiquées qu'occasionnellement, mais elles font partie de l'éventail de possibilités qu'ont les pêcheurs dans l'exercice de leur activité.

Les techniques sont ici présentées comme un système diachronique et complexe issu des interactions entre sociétés, savoirs et milieux. Nous avons choisi de nous appuyer sur trois critères de catégorisation: la zone géographique, le type d'espèces et l'engin de pêche (Figure 2). 
Figure 2 : Principes de catégorisation des techniques mobilisés

\begin{tabular}{l|l}
\hline 1. Les techniques de pêche en étang & \\
\hline 1. 1. Pêcher les poissons et les crustacés & 1.2. Les pêches aux coquillages \\
\hline 1.1.1 Les filets fixes & 1.2.1 L'arselière \\
\hline 1.1.2 Les filets maillants & 1.2.1. La pêche en apnée \\
\hline 1.1.3 Les pêches à l'hameçon & \\
\hline 2. Les techniques de pêche dans les canaux & \\
\hline 2.1. Pêcher les poissons et les crustacés & \\
\hline 2.1.1 Le globe & \\
\hline 2.1.2 Le gangui &
\end{tabular}

\section{Les techniques de pêche en étang}

\section{Pêcher les poissons et les crustacés}

\section{Les filets fixes, un métier qui se fait à la force des bras}

Les engins les plus couramment employés dans les étangs sont les filets fixes. Ils sont constitués d'une paladière, qui est un long filet allant du fond à la surface tenu par des piquets régulièrement espacés. Elle forme ainsi une barrière verticale dont la fonction est de conduire le poisson vers les tours, lieux où se concentrent les verveux, sortes de nasses en forme d'entonnoir dans lesquelles sont piégés les poissons. Quand les poissons y entrent, ils ne peuvent rebrousser chemin. C'est là que le pêcheur les récupère. Le début de la paladière est généralement mis à l'eau de manière perpendiculaire au rivage, en bordure de celui-ci et en allant vers les plus bas-fonds. Pour retirer le poisson de chacun des verveux les pêcheurs en dénouent l'extrémité à l'intérieur de leur embarcation. Les poissons restent vivants et les pêcheurs peuvent, s'ils le souhaitent, relâcher ceux qui ne les intéressent pas ou qui sont trop petits pour être commercialisés. La mise en place de ces engins est relativement longue à effectuer ; ils ne sont entièrement sortis de l'eau que lorsque les filets sont recouverts de salissures car cela influe de manière négative sur l'efficacité de la pêche. 
Figure 3 : Technique de pêche au filet fixe

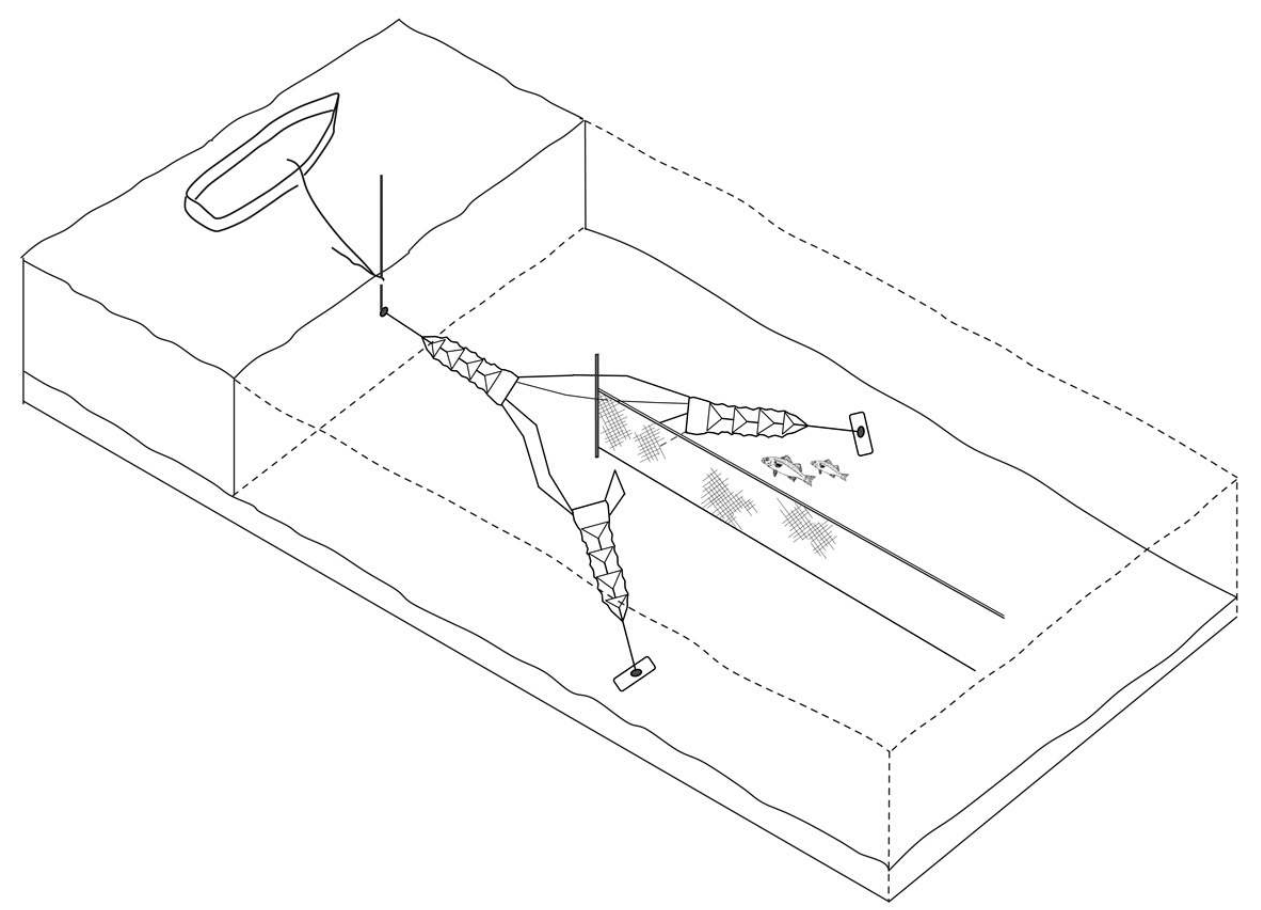

(c) N. Reyes

Figure 4 : Filet fixe vu de dessus

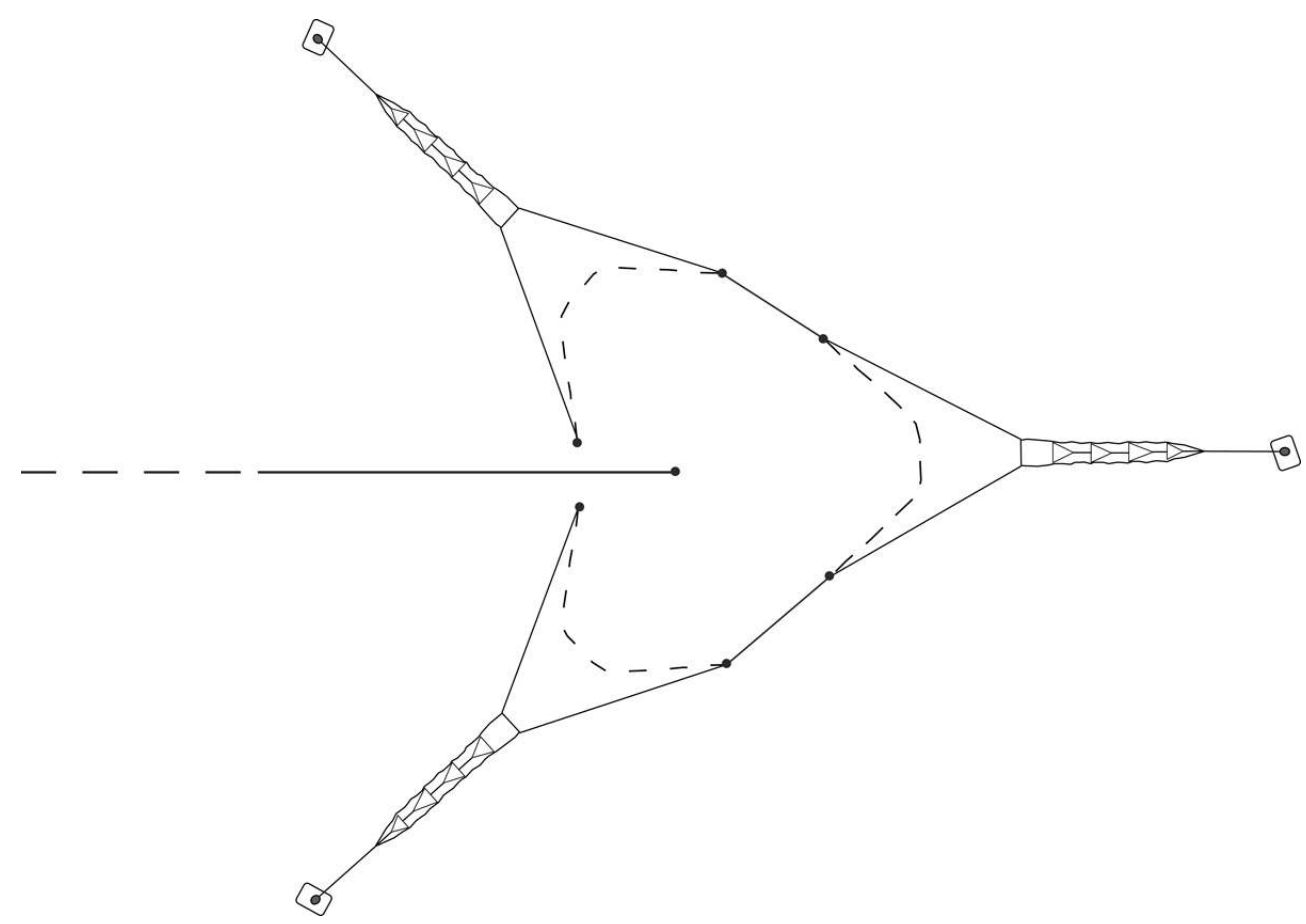

(C) N. Reyes 
Lorsqu'ils parlent de cette technique, les pêcheurs évoquent souvent le temps que prend l'installation de cette pêche:«Tu n'es pas prêt du jour au lendemain. Tu mets environ deux semaines pour être en place ».

Cela est d'autant plus prenant que « c'est lourd comme travail. Une fois que tu es installé, c'est un travail où il faut être régulièrement. Il faut aller voir par roulement les différents filets. Si tu manques d'y aller, tu prends du retard sur leur vérification, l'entretien des suivants et après tu ne t'en sors plus ».

Un autre pêcheur ajoute : «À l'étang, tout le travail, on le fait à la rame et à la perche : c'est comme dans l'ancien temps. Le travail, c'est exactement le même que faisait mon grand-père. Le matériau a évolué... Mais là, tu dors bien. C'est physique ! L'étang c'est un métier qu'il faut aimer ».

Pour évaluer l'influence que peut avoir cette technique sur le milieu, il est donc nécessaire de prendre un compte le temps de latence correspondant à l'installation du pêcheur. De plus, étant donné qu'il s'agit d'un travail difficile, le temps consacré à cette pêche se trouve limité par la capacité physique du pêcheur.

D'un point de vue technique, les espèces pêchées dépendent tout d'abord de la taille des mailles. Les pêcheurs utilisent soit des mailles claires ce qui signifie larges, soit des mailles sèches c'est-à-dire étroites. Les premières permettent de pêcher divers types de poissons comme la daurade (Sparus aurata) ou le loup (Dicentrarchus labrax), tandis que les secondes capturent plutôt les anguilles (Anguilla anguilla) ou le joël (Atherina (Hepsetia) boyeri). L'autre aspect technique qui influe sur la nature des captures est la forme de la pose. En effet, les filets fixes peuvent être disposés de différentes manières qui correspondent pour les pêcheurs à autant de techniques de pêches différentes. Selon les époques de l'année la forme de pose varie. De plus, chacune a une influence différente sur le milieu car certaines formes permettent de cibler une espèce plutôt qu'une autre. En effet, un pêcheur explique que dans les filets à mailles sèches, les poissons se prennent très peu en raison des mailles serrées, car ils voient le filet et l'évitent.

\section{Les filets maillants : " effrayer le poisson " ou " le laisser se faire prendre"}

«Les filets [maillants] n'ont jamais été les engins dominants de la pêche lagunaire

[...] il n'en reste cependant pas moins qu'ils aient, à toute époque, été largement utilisés » (Giovannoni 1995 : 124).

Les filets maillants regroupent deux types de filets : les filets droits et les trémails.

Les filets droits, appelés aussi nappes ou pièces, sont formés d'une seule nappe de filet rectangulaire. Elle est faite de nylon, souvent appelé crin par les pêcheurs du fait de sa clarté. Ces engins sont de hauteur très variable selon la profondeur de la zone de pêche. En étang, ils peuvent mesurer de un à dix mètres et créer des barrières allant de la surface au fond. Néanmoins, ces engins sont considérés comme très sélectifs car ils permettent de cibler une taille de poisson spécifique. Ils permettent de capturer divers types de poissons, les mêmes que ceux précédemment cités à l'exception des anguilles. C'est ensuite la zone de pose qui détermine l'espèce qui va être ciblée.

Les trémails sont formés de trois nappes de filets: une bande de maille fine (que l'on appelle ici le lis) insérée entre deux bandes de mailles plus larges (les armals). Ces engins capturent le poisson par boursage, c'est-à-dire que le poisson passe la première maille, mais il est arrêté par la seconde pour se retrouver enfin pris, dans une bourse, en dépassant la troisième nappe. À la différence des pièces, les poissons de plus grande taille 
se prennent dans le filet en plus des poissons correspondant à la taille de la maille. Cette technique permet, de plus, de prendre les poissons plats tels que les soles (Solea vulgaris). Il semblerait qu'ils soient plus efficaces que les pièces, cependant, la hauteur de ces filets ne dépasse pas quatre mètres. En effet, les algues s'y prennent très facilement et cela demande un travail trop important pour les nettoyer. Ils sont donc utilisés avec parcimonie.

Figure 5 : Technique du filet maillant

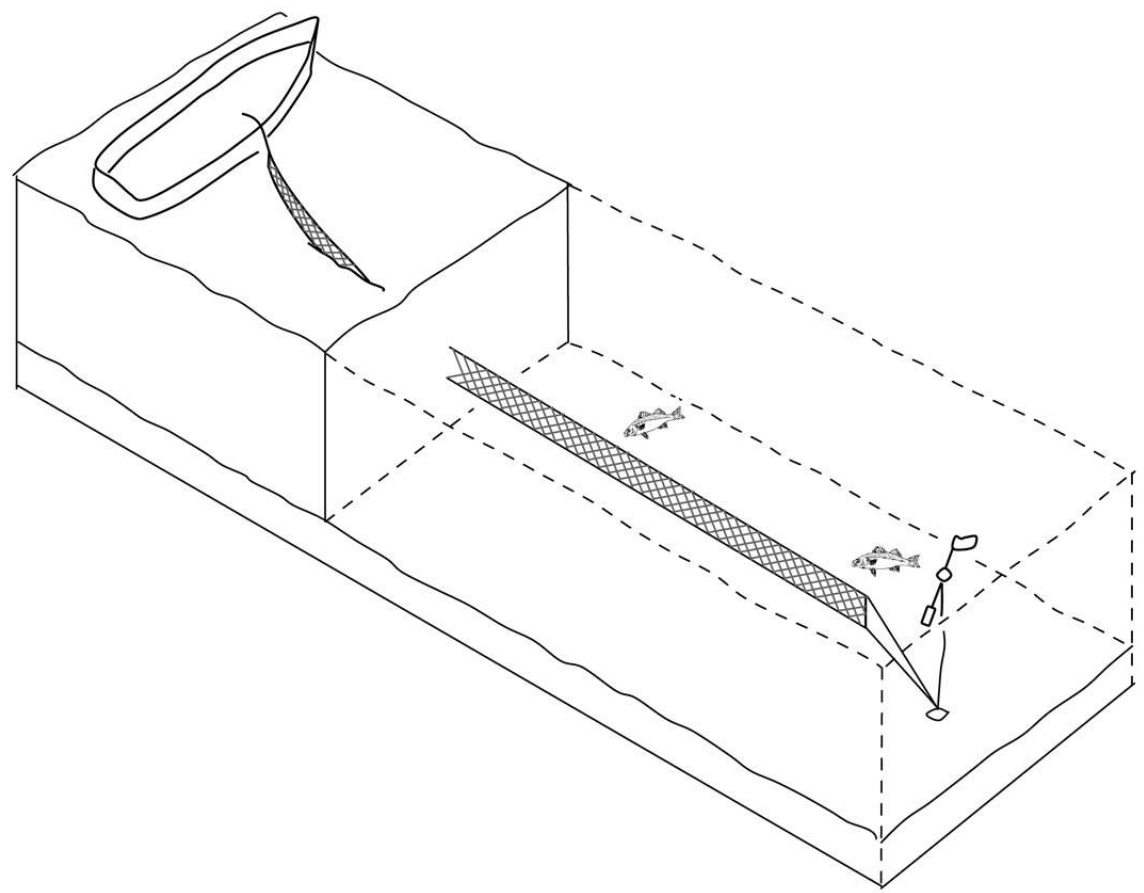

(C) N. Reyes 
Figure 6a : Capture au filet droit

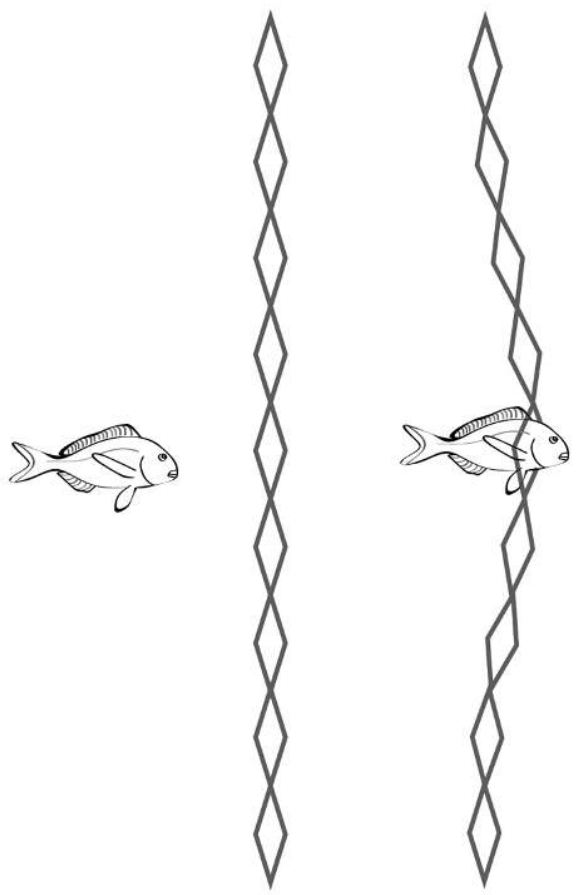

(C) N. Reyes

Figure 6b : Capture au filet trémail par boursage
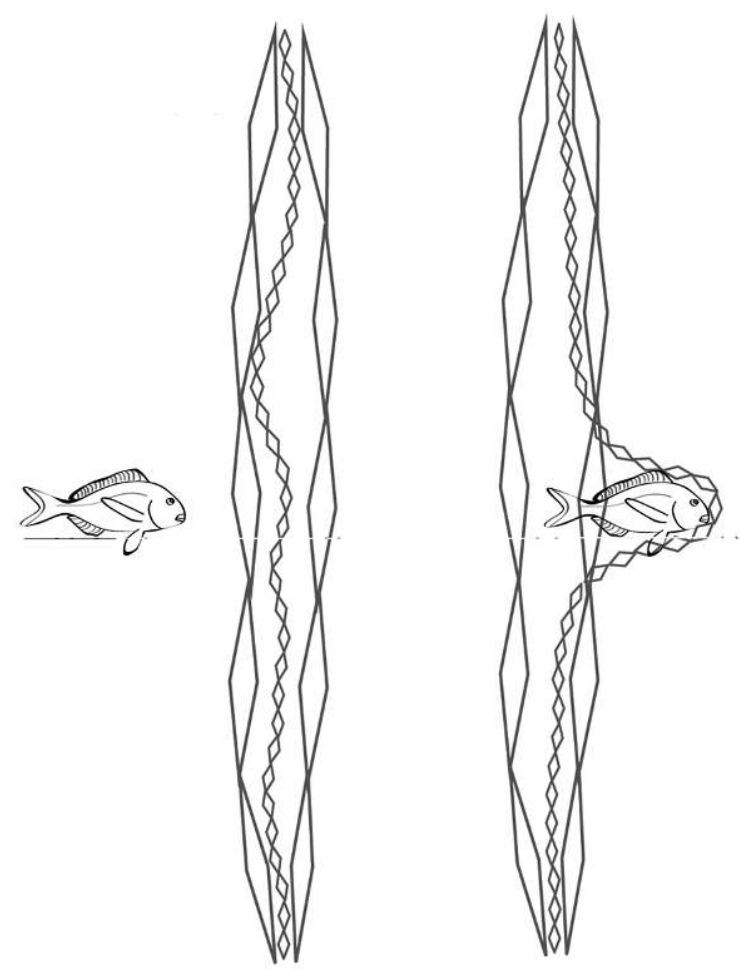

(c) N. Reyes

Revue d'ethnoécologie, 7 | 2015 
40

La battue, consiste à encercler le poisson. Le pêcheur se place au centre et bat les eaux afin d'orienter les déplacements des poissons pour qu'ils se prennent dans le filet qui les entoure. Auparavant, cette pêche était pratiquée à deux. Un pêcheur raconte qu'autrefois cette technique "on la faisait à l'oreille. On écoutait le poisson chaouper (faire des remous). On prenait le temps de l'encercler sans faire de bruit. Mais aujourd'hui... on ne devrait pas, mais... c'est un métier qu'on fait, seul, au moteur. On essaye d'aller vite mais ça fait du bruit, alors le poisson fuit et donc on le prend peu... ».

41 Le champ de l'anthropologie des techniques considère que la battue est une technique active à la différence de la maïdane. Cette dernière consiste à mettre à l'eau des filets, souvent en baguechan c'est-à-dire en effectuant des zigzags, en les laissant de plusieurs heures à quelques jours selon les espèces recherchées. Elle est considérée comme passive de ce point de vue. Dans ce cas, on parle alors de filet dormant. Les filets utilisés pour la technique à la maïdane sont souvent plus longs que les filets à battue, où l'utilisation de filet avec peu de longueur permet davantage de mobilité. De plus, du fait de la différence de durée de mise à l'eau de l'engin de pêche, ces techniques vont avoir une influence différente sur le milieu. En effet, en laissant à l'eau des filets pendant plusieurs jours, il arrive que les pêcheurs remontent des espèces qui sont abimées et qu'ils ne peuvent commercialiser. L'effort de pêche ${ }^{17}$ sur le milieu est donc différent.

\section{Pêcher à l'hameçon pour cibler une espèce}

Parmi les engins de ligne utilisés, on trouve les cros. Il s'agit d'une ligne simple, pourvue d'un ou deux hameçons, maintenue à la surface par un flotteur et sur le fond par une pierre. Les pêcheurs mettent à l'eau plusieurs cros et utilisent généralement un appât vivant. Cet engin permet surtout de pêcher les espèces carnivores telles que le loup ( Dicentrarchus labrax) et la grosse anguille verte (Anguilla anguilla). Cet engin ne permet de capturer qu'une seule espèce à la fois. Cependant, étant de petite dimension, il peut être disposé en des endroits choisis. 


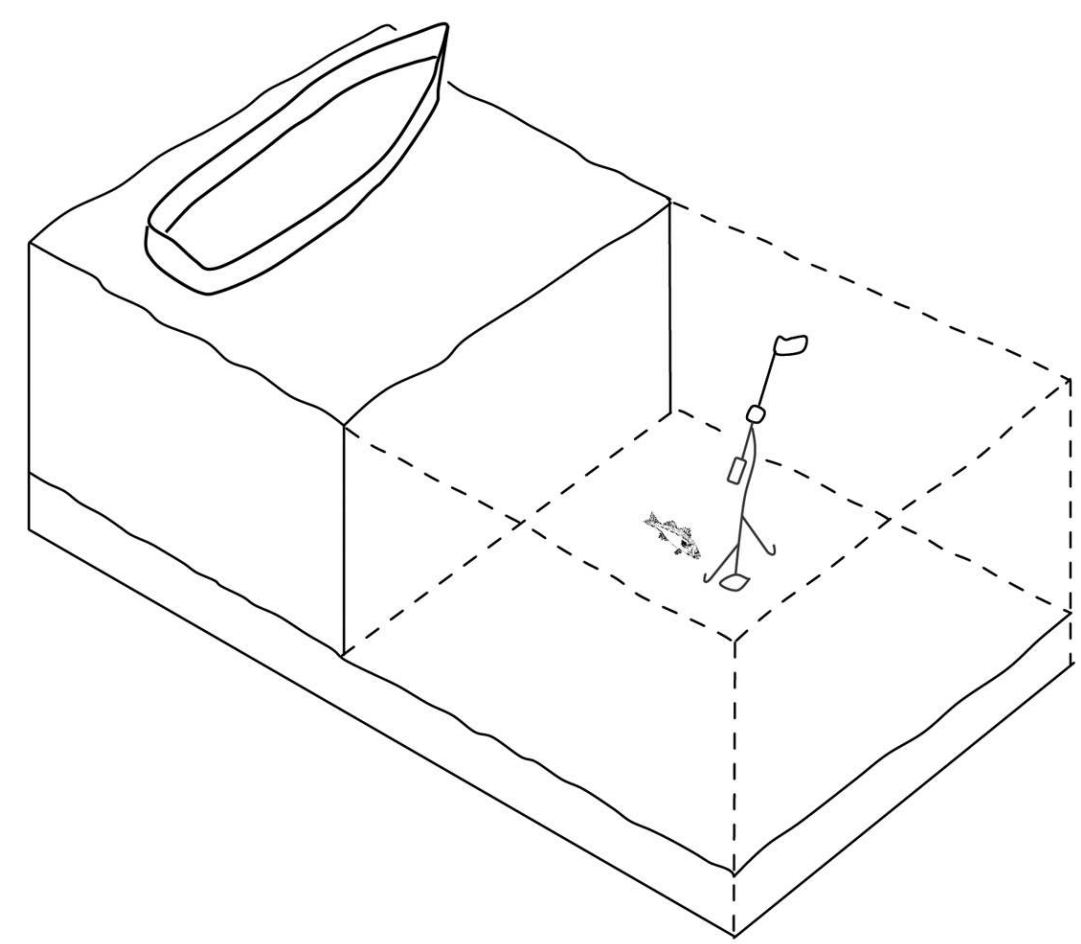

(c) N. Reyes

Une des autres techniques de pêche à l'hameçon exercée est la palangre. Il s'agit d'un engin de pêche passif composé d'une ligne mère sur laquelle se trouvent des avançons, c'est-à-dire des lignes qui se finissent par des hameçons, placés à intervalles réguliers. Cet engin selon qu'il est sur le fond ou entre deux eaux ne cible pas les mêmes espèces. Il est souvent qualifié par les pêcheurs de sélectif. Le montage de l'engin (diamètre de la ligne mère et des avançons, longueur et distance entre les avançons, etc.) et l'appât que va choisir le pêcheur orientent les prises possibles vers une espèce cible. Cependant, la pêche n'étant pas une activité infaillible, des espèces non recherchées s'y prennent parfois. Par ailleurs, cette technique permet de capturer des poissons vigoureux qui cherchent à se nourrir. 


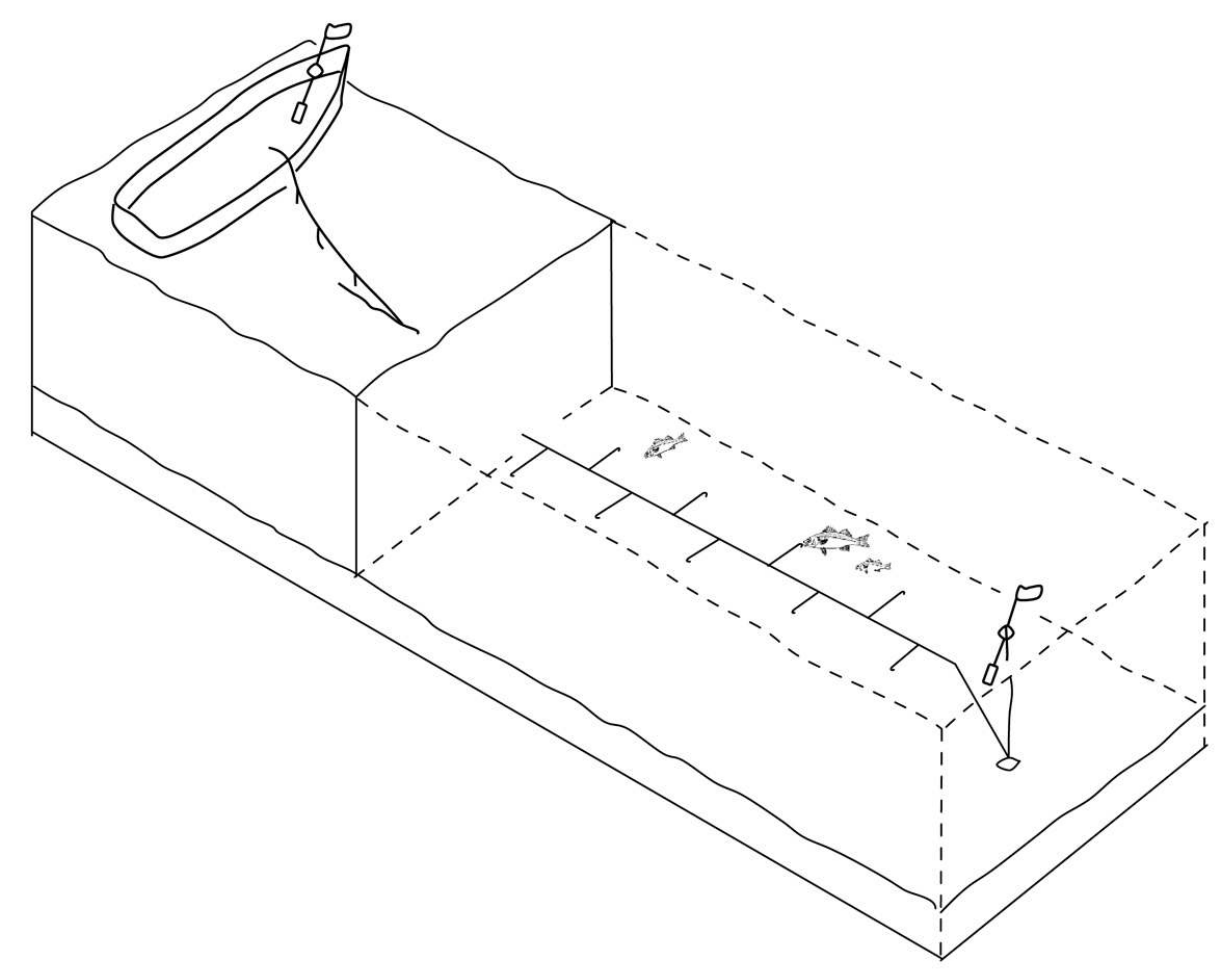

(c) N. Reyes

\section{Les pêches aux coquillages}

\section{L'arselière, « un travail de forçat "}

44 Autrefois très pratiquée, l'arselière est une technique de pêche qui tombe aujourd'hui en désuétude. Cet engin s'apparente à un râteau de très grande taille, au manche de sept à huit mètres, comportant en son extrémité un filet en forme de poche qui sert de récolteur. Il est manœuvré par l'homme qui se tient debout sur sa barque. Un pêcheur dira, à propos de l'arselière que: "c'est un travail de forçat, que même celui qui l'a inventé, il le ferait pas!». 


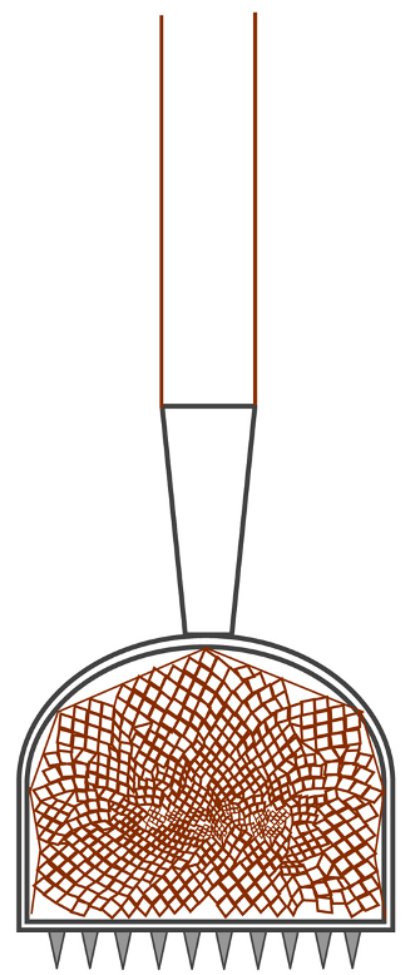

(c) N. Reyes

\section{Être en bonne forme physique, une condition pour exercer la pêche en apnée}

Cette technique vise principalement les palourdes (Ruditapes decussatus). Le plongeur est muni d'une fourchette aux dents longues d'environ 12 à 15 centimètres qui lui permet d'extraire les coquillages enfouis. En effet, « la palourde, tu la repères parce qu'elle fait des trous dans le sable qui lui permettent de respirer et de manger » rapporte un pêcheur qui a pratiqué cette technique pendant plus de vingt ans. Il ajoute, «c'est pas trop compliqué mais pour pratiquer [cette technique] il faut avoir une bonne vue et être en forme... parce que... tu fais que monter pour prendre ta respiration et redescendre. Moi, je ne sortais jamais faire la fête. Sinon, je passais à côté des trous sans les voir ».

Mis à part les palourdes, cette technique permet aussi de récolter le naissain de moule ( Mytilus galloprovincialis) ou d'huîtres (Crassostrea gigas) ainsi que toutes autres sortes de mollusques.

\section{Les techniques de pêche dans les canaux}

Pêcher les poissons et les crustacés

Deux techniques de pêche sont principalement pratiquées dans les canaux. La première se rapporte aux techniques de filets soulevés et la seconde aux techniques de filets fixes. 


\section{Le globe, une pratique de nuit} à l'autre du canal des étangs. Un des bords de l'engin est hors de l'eau et l'autre partie est submergée. Pour le pêcheur «avec cette technique, tu travailles avec le courant et les sens de migration du poisson ». Ainsi, lorsqu'un banc de loup, principale espèce ciblée, s'est engagé sur le filet, on relève l'autre bord au moyen de tourniquets de manière à ce qu'il se trouve pris au piège.

Figure 10 : Technique de pêche au globe

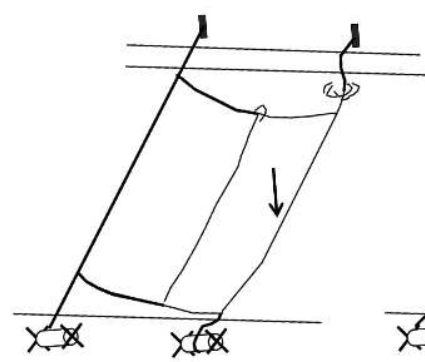

1

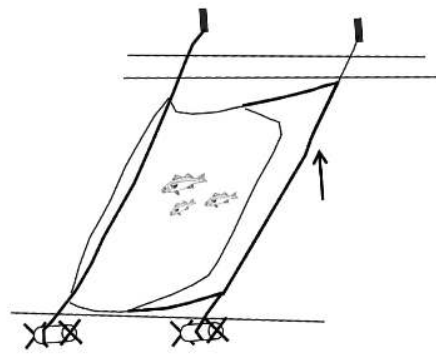

3

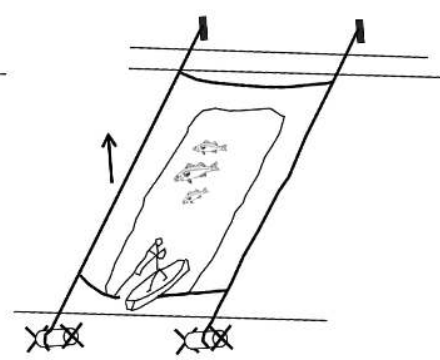

4

(C) N. Reyes

Ala différence des autres techniques, cette pêche est pratiquée durant la soirée et la nuit. Les pêcheurs procèdent plusieurs fois à la levée et à l'abaissement de l'engin. En effet : « le globe, tu restes éveillé toute la nuit. Bon, on ne la fait pas tous les jours... il faut qu'il y ait un gros coup de mistral à l'automne. Quand il y a beaucoup de vent et un coup de froid, tu peux être sûr que tu vas pêcher ».

Ensuite, comme pour les filets fixes, les espèces prises dépendent de la taille des mailles que le pêcheur utilise. Cependant, la technique du globe tend à être davantage pratiquée avec de larges mailles. À ce propos, un pêcheur dira : «c'est un métier fatigant alors s'il y a moins de surface, ça se lève mieux ».

Il précise: "C'est une pêche qui se faisait beaucoup avant. Elle est spéciale cette technique. Tu vois, mon père m'apprend encore des choses sur le globe ».

53

Revue d'ethnoécologie, 7| 2015 


\section{Le filet fixe pour les petites espèces}

\section{de deux ailes qui rejoignent les berges et d'une grande nasse (poche) centrale dans laquelle} sont capturées les espèces.

Figure 11 : Technique de pêche au gangui
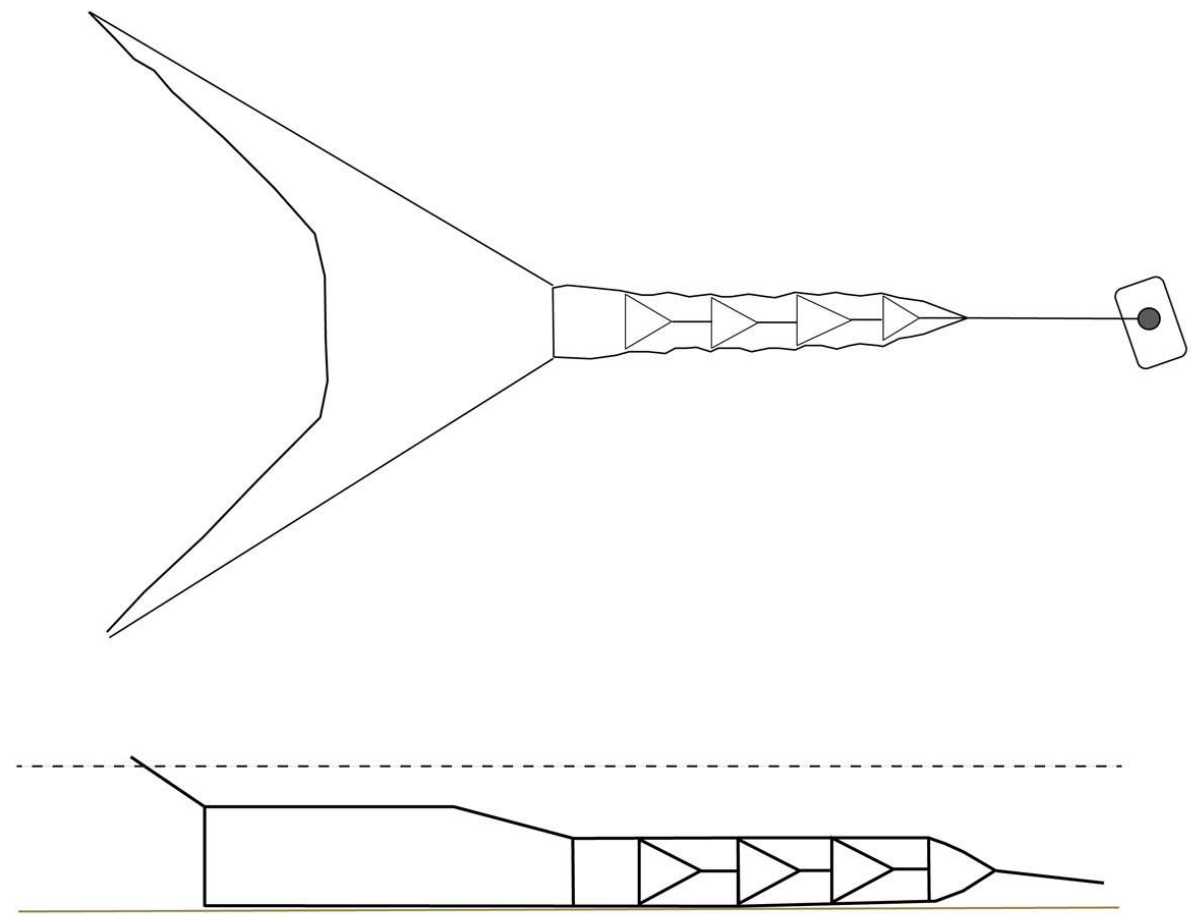

\section{(c) N. Reyes}

Cette technique permet de capturer essentiellement du joël (Atherina (Hepsetia) boyeri) et de la sivade ou crevette grise (Crangon crangon). Le pêcheur le place le soir et le relève quelques heures après. Certaines conditions météorologiques spécifiques (par exemple vents de mistral) sont nécessaires pour exercer cette technique. Celles-ci ne sont réunies que pour une période relativement courte (par exemple une quinzaine de jours).

Il n'est pas question de faire un inventaire exhaustif des techniques de pêches pratiquées par les pêcheurs aux " petits métiers » dans les étangs. L'idée est plutôt de montrer que les pêcheurs disposent d'un éventail de techniques assez larges. Les interactions que les pêcheurs auront avec le milieu varient donc selon la technique qu'ils choisiront d'exercer. En effet, nous avons montré que chaque technique correspond pour le pêcheur à une manière spécifique de travailler. En cela, les affects des pêcheurs pour une technique, l'expérience qu'ils ont de celle-ci les pousseront à utiliser un engin plutôt qu'un autre. Ainsi, les techniques ne sont pas toutes à considérer sur le même plan. De plus, nous avons cherché à montrer que pour les pêcheurs, les techniques sont associées à la recherche de certaines espèces en particulier. En effet, chaque espèce possède un comportement spécifique auquel le pêcheur doit s'adapter en utilisant chaque 
fois une technique appropriée (idée que nous dénommerons ci-après par l'expression: " couple engin-espèce »). Comme l'explique très bien un pêcheur de Palavas-les Flots :

«Un filet qui pêche de tout, pour nous, ça n'existe pas ».

Ainsi, à la diversité des espèces ciblées, correspond une diversité de techniques. De ce fait " chaque technique, et plus particulièrement chaque engin, doit être considéré comme le fruit d'un ensemble de savoirs orientés vers l'efficacité [...]. Devant ruser avec la défiance de chaque espèce, le principe qui induit les formes de chaque pratique, de chaque engin, doit être compris comme le résultat matériel de la confrontation de deux intelligences: celle de l'homme et celle des poissons » (Giovannoni 1995).

Néanmoins, cette présentation ne doit pas conduire le lecteur à penser que ces techniques ont existé de tout temps. En effet, celles-ci évoluent notamment avec l'apparition de nouveaux matériaux, les procédés d'innovation et les variations du marché local, national et international. La permanence d'une technique dépend du prix de vente des captures, de sa capacité à capturer les espèces cibles et du coût de sa mise en œuvre (matériel, main d'œuvre, énergie déployée). Ces éléments doivent s'ajuster afin que la technique soit suffisamment concurrentielle comparativement aux autres techniques existantes. On conçoit donc que l'adoption par les pêcheurs d'une technique dépend notamment du fait qu'elle correspond à un certain niveau d'efficacité et de rentabilité. Une technique est donc le fait d'ajustements et d'évolutions de la part du pêcheur qui peuvent l'amener à modifier ses modes d'interaction avec le milieu.

\section{Des savoirs associés à des milieux spécifiques : une appropriation contrastée de l'espace de pêche}

Alors que l'on pourrait penser que la proximité géographique tend à produire des pratiques similaires, cela n'est pas le cas. Une approche fine montre que chaque technique est adaptée localement. En effet, les pêcheurs professionnels associent à chaque étang une manière de travailler totalement différente. Cette interaction entre territoires et savoirs est au cœur des pratiques des pêcheurs.

61 En mettant en regard les caractéristiques écologiques des différents étangs on constate d'importantes différences. La profondeur, le degré de renouvellement des masses d'eau, le sens des courants, la couleur, la conformation particulière des fonds et la position géographique vis-à-vis des vents sont essentiels et vont influer sur les activités humaines d'exploitation du milieu lagunaire. Pour comprendre ce lien, l'exemple le plus évident est celui de la profondeur du milieu, puisque selon cette donnée, le pêcheur devra ajuster la hauteur de son matériel de pêche. Ainsi, à l'étang de Thau dont la profondeur maximum est de 30 mètres répond le complexe lagunaire des neuf étangs palavasiens dont les deux étangs les plus profonds atteignent respectivement 2 mètres et 1,5 mètre tandis que les autres sont d'en moyenne 0,6 mètre. Les profondeurs de chacun des étangs n'étant pas les mêmes, les hauteurs d'un même engin ne sont, par conséquent, pas les mêmes non plus.

Par ailleurs, les entretiens montrent que pour les pêcheurs, la nature et la quantité d'une même espèce peuvent varier d'un étang à l'autre. Un pêcheur de Palavas-les-Flots, ayant plus de 30 ans d'expérience et issu d'une famille de pêcheurs, rapporte qu'entre professionnels ils disent que «pour le poisson l'étang de Vic c'est mieux que l'étang de l'Or. Mais bon, pour [la présence des] poissons, ça dépend des étangs, du temps qu'il fait, 
de là où est entré le poisson, [...] s'il y a eu de l'herbe, s'il a fait chaud. Après c'est la nature! ».

La présence et l'abondance des espèces n'est donc pas une donnée fixe. On comprend donc que selon ces critères, certaines techniques de pêche seront davantage exercées dans certains étangs que dans d'autres, et ce, même si une technique se trouve pratiquée en divers endroits. En outre, certaines techniques ne sont présentes que très localement, c'est le cas de la pêche en plongée, activité qui n'est présente que sur l'étang de Thau.

En interaction quasi quotidienne avec le milieu, les pêcheurs accumulent des observations fines et disposent d'un suivi continu de l'évolution des milieux qu'ils exploitent. Par ailleurs, alors que la littérature sur le complexe lagunaire de Palavas-lesflots distingue neuf étangs qui se succèdent d'ouest en est comme suit : l'étang de l'Or, Vic, Mourre, Pierre-blanche, Arnel, le Prévost, le Méjean, l'étang du Pérol ou la falaque et le Grec, pour les pêcheurs aux "petits métiers » de Palavas-les-Flots ce complexe est plutôt composé de onze étangs. À ceux précédemment cités s'ajoutent l'étang de l'Aïdole et $d u$ Gran canaú. Cette différence dans le nombre d'appellations est révélatrice d'une différence de conception de l'espace entre les acteurs. En effet, le fait de nommer correspond au fait de donner une identité propre et des contours à ce qui fait l'objet de pensée (Lévi-Strauss 2008 [1962]). Si les pêcheurs professionnels ont attribué au complexe lagunaire palavasien des appellations supplémentaires, c'est qu'ils considèrent ces espaces comme correspondant à des réalités différentes.

Cette différenciation fine des espaces tient aussi au fait que pour les pêcheurs il existe, au sein même des étangs, des zones qui sont plus favorables que d'autres. Celle-ci prend sa forme la plus évocatrice dans le cadre du tirage au sort des zones de pêche pour la technique du filet fixe. Le tirage se déroule en deux phases: la première consiste en ce que chacun des pêcheurs passe à tour de rôle tirer un numéro de passage. La seconde phase repose sur le numéro pioché qui permet de choisir les zones fixes, appelées postes par les pêcheurs. Par ordre croissant, chacun des pêcheurs défile devant le tableau pour écrire son nom devant le poste qu'il a choisi. Le nombre de postes étant supérieur au nombre de participants, ceci donne lieu à un second passage dont l'ordre est inversé. Ce tirage présente un enjeu pour le pêcheur, car de la qualité des postes qu'il obtient dépend le niveau de revenu qu'il fera au cours de l'année. Le tirage au sort est organisé chaque année par "la Prud'homie des pêcheurs", une institution professionnelle locale qui se charge de la gestion de l'activité ${ }^{19}$. Il s'inscrit dans une démarche de répartition équitable de l'espace. Il permet d'éviter que les meilleures zones ne soient accaparées par certains et que des conflits surviennent entre pêcheurs. Chacune d'elles est dénommée de manière précise. Sur l'étang de Thau, par exemple, ces dénominations ont des origines aussi bien terrestres que marines: Dégol, Sèche, Terre rouge, Caraman, Canal de Sète, Pont Carnot, Tombant, Brameur, Grosse bouée, Nord chenal, Bout du Barrou, Pointe de la Mousse, Barrou, Alfangade, Lafarge (Giovannoni 1995).

Les tirages de la Prud'homie de Palavas-les-Flots et de celle de Sète suivent le même processus. Cependant, la multiplicité des étangs palavasiens oblige les pêcheurs à faire le choix de l'étang dans lequel ils veulent tirer leur poste de pêche. À la différence de la conception spatiale des postes de pêches à l'étang de Thau qui sont prévus pour un engin, ces postes-là sont bien plus étendus. À l'étang de Vic, par exemple, ils couvrent une surface d'environ 600 mètres de long pour 350 mètres de large. Ainsi, ces postes permettent de placer près de 10 filets fixes. 

Outre le fait de procéder à une répartition de l'espace, le tirage au sort limite l'exploitation d'une zone à un seul individu. Il permet au pêcheur de s'approprier temporairement un espace.

\section{Conclusion}

Cette étude de cas s'est volontairement concentrée sur les activités de pêche en étang afin de montrer l'étendue de la diversité des pratiques existantes. La diversité des savoirs, des perceptions et des manières de travailler des pêcheurs aux "petits métiers " s'avère d'autant plus accentuée qu'elle est associée à un contexte ultra-localisé relatif au milieu et aux ressources exploitées.

69 Toutefois, les activités de pêche lagunaire ne correspondent qu'à un pan des activités exercées par les pêcheurs aux " petits métiers » languedociens. En effet, l'activité de ces pêcheurs s'exerce également sur le milieu maritime, et cela aussi bien sur la bande côtière qu'au large. Ainsi l'étendue de l'hétérogénéité que recouvre la catégorie " petits métiers » est encore plus ample.

Figure 12 : Bateaux armés à la pêche aux « petits métiers » en mer, Sète

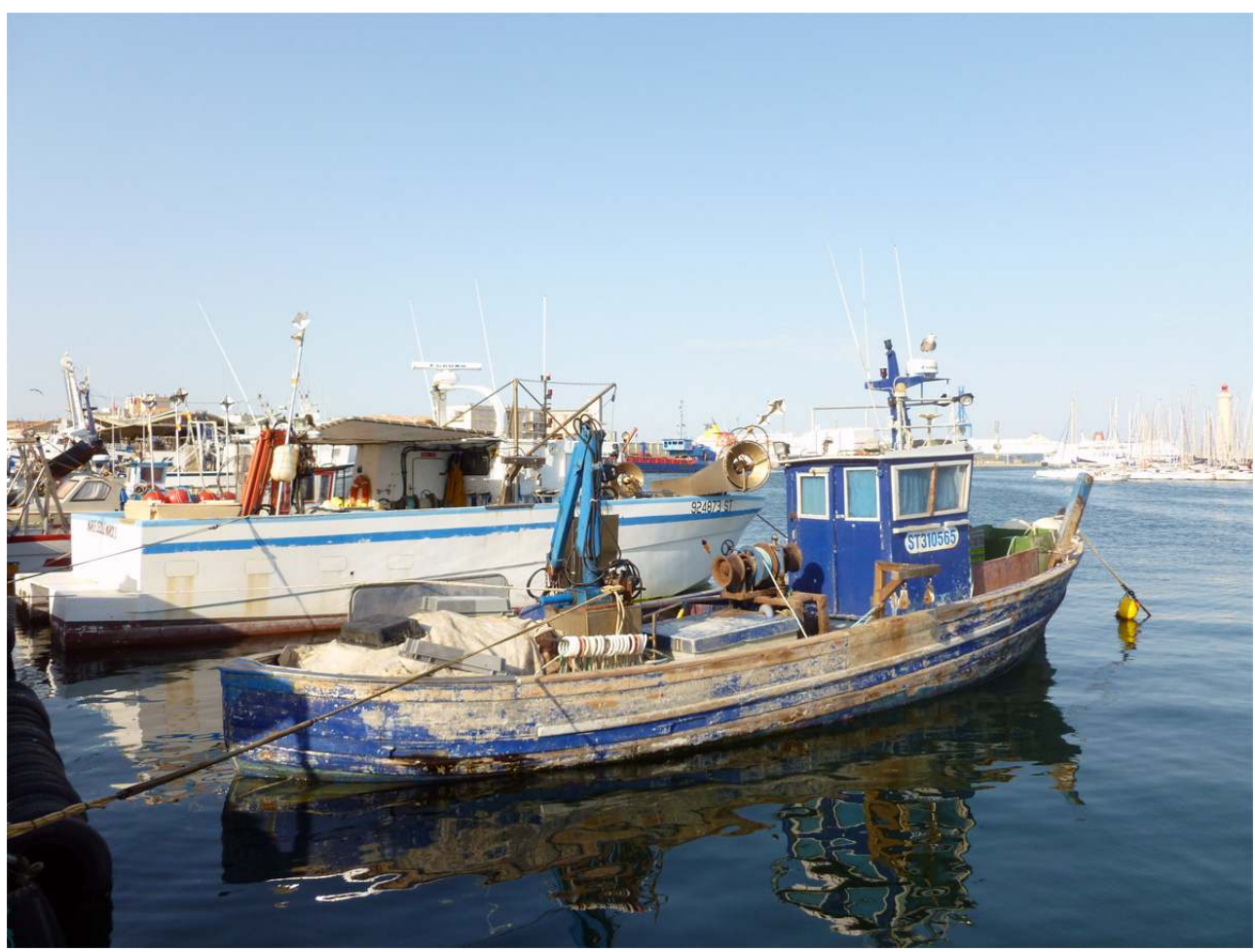

(C) N. Reyes

À ce titre, la description des pêches dans le golfe de Marseille réalisée par Paul Gourret à la toute fin du XIX ${ }^{e}$ siècle atteste là aussi d'une importante polyvalence des techniques de pêches côtières exercées sur le milieu maritime. Il s'applique à décrire une quarantaine de techniques différentes, impliquant des engins variés: quatre types d'hameçons et lignes, quatre sortes de casiers, deux techniques de harpons, douze sortes de filets traînants, cinq filets dérivants et douze types de filets fixes. Si P. Gourret rapporte une 
rivalité des métiers entre les partisans des «arts fixes et flottants» et ceux des «arts dragueurs ", il évoque aussi la diversité des techniques employées par les patrons pêcheurs: «Le plus souvent, les patrons thysairé [sorte de trémail] n'exercent pas exclusivement cet art fixe et se livrent, suivant les époques et les circonstances, à d'autres pêches. Les uns ont des battudes et des battudons [types de filets fixes] et même des palangres, les autres des thonaires et des sardinaux [types de filets dérivants], d'autres un bourgin [sorte de senne], une siouclétière ou une mugelière [types de filets fixes], quelques-uns un gangui à la voile [chalut]. » (Gourret 1934 [1894] : 237).

71 Ainsi, l'élément principal qui distingue la pratique des pêcheurs aux « petits métiers » des autres acteurs de la pêche est la diversité des techniques employées, qui se développent dans divers écosystèmes aquatiques. En effet, elle est rarement restreinte à l'exploitation d'un seul milieu et on constate qu'au cours de son parcours professionnel, le pêcheur peut faire preuve de mobilité et exercer son activité dans plusieurs de ces différents milieux (Reyes 2012). Ainsi, une dénomination unique pour l'activité de pêche aux "petits métiers » donne l'illusion d'une catégorie homogène, là où en réalité sont réunies des pratiques de pêche extrêmement variées. C'est donc cette diversité qui constitue, pour les pêcheurs, la caractéristique fondamentale de cette activité.

La revue des différentes expressions employées dans les législations nationale et européenne pour évoquer les "petits métiers » de la pêche et l'analyse des rapports des pêcheurs aux milieux et à la ressource qu'ils exploitent, nous permettent de proposer des orientations pour une meilleure définition. Nous choisissons de conserver l'expression "petits-métiers" dans la mesure où l'usage du pluriel suggère l'hétérogénéité intrinsèque qui la compose. Ce choix est, de plus, en accord avec la manière dont les pêcheurs dénomment l'activité qu'ils exercent et avec la démarche inductive que nous adoptons. Six paramètres sont ainsi choisis, correspondant à l'activité " petits métiers " telle que considérée par les pêcheurs eux-mêmes :

1. La polyvalence de l'activité de pêche (au sens de l'association fine du «couple enginespèce $»)$;

2. L'absence d'arts traînants (à l'exception des dérogations qui figurent dans l'arrêté du 13 mai 2014 portant adoption de plans de gestion pour les activités de pêche professionnelle à la senne tournante coulissante, à la drague, à la senne de plage et au gangui en mer Méditerranée par les navires battant pavillon français);

3. La durée de sortie en mer inférieure ou égale à 24 heures;

4. La déclaration du propriétaire du navire en tant que "patron-embarqué »;

5. La zone de navigation s'étendant des eaux abritées (telles que rades non exposées, bassins ou étangs d'eau salée, etc.) jusqu'à la bande maritime des 20 milles marins (caractéristique qui témoigne d'un ancrage territorial fort) ;

6. Le navire ne dépassant généralement pas les 12 mètres de longueur mais pouvant s'étendre à une longueur maximale de 15 mètres (Ifremer 2007).

Cette proposition multidimensionnelle inclut des caractéristiques techniques (taille du navire et engins autorisés), de navigation (distance des côtes et temps de sortie en mer) et fiscales (statut d'imposition des sociétés artisanales). Nous noterons qu'elle rejoint les critères suggérés par l'Ifremer dans un rapport de 2007 pour une définition utile et opérationnelle des petites pêches européennes ${ }^{20}$. Elle est, de plus, issue de la compilation et de la recomposition des différentes expressions exposées en première partie. Cette proposition suggère les bases d'une redéfinition des critères pour lesquelles le premier critère est fondamental tandis que les suivants admettent une certaine flexibilité. 
74 et de poser les critères de référence qui, selon les pêcheurs, correspondent à une vision cohérente de ce qu'est l'activité de pêche aux « petits métiers ».

Remerciements à Christian Chaboud, économiste des pêches à l'IRD de Sète (UMR MARBEC) et à Bertrand Cazalet, consultant indépendant, juriste en droit public et droit international.

\section{BIBLIOGRAPHIE}

Arrêté du 19 décembre 1994 portant réglementation technique pour la pêche professionnelle en Méditerranée continentale.

Arrêté du 13 mai 2014 portant adoption de plans de gestion pour les activités de pêche professionnelle à la senne tournante coulissante, à la drague, à la senne de plage et au gangui en mer Méditerranée par les navires battant pavillon français.

Article L 931-2, L IX, T III, S 1, Sous-section 1 - « Société de pêche artisanale », Code rural et de la pêche maritime.

Bahuchet S. 1986 - Ethnoécologie comparée des pygmées Aka et des villageois de Ngando de la Lobaye (RCA). Écologie Humaine 4 (2) : 3-18.

Bahuchet S. 1992 - Esquisse de l'ethnoichtyologie des Yasa du Cameroun. Anthropos 87 : 512-520.

Campillo A. (Ed.) 1992 - Les pêcheries françaises en Méditerranée continentale. Synthèse des connaissances. Perspectives. Sète, Ifremer, DRV-86.0004/RH/Sète, 206 p.

Cury P. \& Miserey Y. 2008 - Une mer sans poissons. Paris, Calmann-Lévy, 283 p.

Décret 93-33 du 8 janvier 1993 relatif au permis de mise en exploitation des navires de pêche pris pour l'application de l'article 3-1 du décret du 9 janvier 1852 modifié sur l'exercice de la pêche maritime.

Décret 93-1342 du 28 décembre 1993 relatif aux conditions d'exercice des fonctions de capitaine et d'officier à bord des navires de commerce, de pêche et de plaisance.

Décret 2011-697 du 20 juin 2011 définissant la notion de petite pêche côtière mentionnée au $6 \mathrm{du}$ II de l'article 262 du code général des impôts.

Décret 2012-554 du 23 avril 2012 modifiant le décret n²007-1377 du 21 septembre 2007 portant diverses dispositions relatives aux titres de formation professionnelle maritime.

Dupouy A. 1955 - La pêche maritime et le pêcheur en mer. Paris, Armand Colin, 216 p.

Farrugio H. \& Le Corre G. 1985 - Les pêcheries de lagune en Méditerranée. Définition d'une stratégie d'évaluation. [En ligne : http://archimer.ifremer.fr/doc/00075/18608/]

Féral F. 1980 - La prud'homie des pêcheurs de Palavas. Étude de la mort d'une institution. Lyon, Publications Périodiques Spécialisées, $314 \mathrm{p}$.

Féral F. 1987 - Un hiatus dans l'administration et la politique des pêches maritimes : les prud'homies de pêcheurs en Méditerranée. Norois 133-135 : 355-369. 
FranceAgriMer 2013 - Les filières pêche et aquaculture en France. Pêche et aquaculture. Production, entreprises, échanges, consommation. Les cahiers de FranceAgriMer, 36 p.

Frangoudes K. 1995 - Changing fisheries management institutions in the French Mediterranean. Workshop in Political Theory and policy analysis $10 \mathrm{p}$.

Geistdoerfer A. 1974 - Savoir et techniques des pêcheurs des îles de la Madeleine (Québec) ou de quelques moyens de s'approprier la mer et ses ressources. Journal d'agriculture tropicale et de botanique appliquée XXI (7-8-9) : 169-204.

Geistdoerfer A. 1977 - Connaissance techniques et patrimoine maritime. Études Rurales 65 (janv.mars) : 49-58.

Geistdoerfer A. 1985 - S'approprier la mer aux îles de la Madeleine, Québec, et à Saint-Pierre-etMiquelon. Anthropologie Maritime, CETMA, 2 : 31-40.

Giovannoni V. 1995 - Les pêcheurs de l'étang de Thau. Écologie humaine et ethnologie des techniques. Paris, L'Harmattan, 283 p.

Gourret P. 1934 [1894] - Les pêcheries et les poissons de la Méditerranée (Provence). Paris, Baillière, $360 \mathrm{p}$.

Guillou A., Lespagnol P. \& Ruchon F. 2002 - La pêche aux petits métiers en Languedoc-Roussillon en 2000-2001. Ifremer, Centre halieutique méditerranée et tropical, Laboratoire Ressources Halieutiques, $108 \mathrm{p}$.

Ifremer 2002 - Réseau de Suivi Lagunaire du Languedoc-Roussillon, Rapport 2001, 343 p.

Ifremer (coord.) 2007 - Small-Scale Coastal Fisheries in Europe, Final report of the contract No FISH/2005/10, 447 p.

Jacquet J. \& Pauly D. 2008 - Funding priorities: big barriers to small-scale fisheries. Conservation Biology 22 :832-5.

Lecou R. 2010 - Rapport d'information sur les perspectives d'une pêche durable en Méditerranée. Paris, Commission des affaires européennes, $88 \mathrm{p}$.

Lenthéric C. 1876 - Les Villes mortes du Golfe de Lyon, Illiberris - Ruscino - Narbonne - Agde - Maguelone - Aigues-Mortes - Arles - Les Saintes-Maries-de-la-mer. Paris, Plon, 524 p.

Lévi-Strauss C. 2008 [1962] - La Pensée sauvage. In : CEuvres Paris, Gallimard/Pléiade : 553-872.

Malinowski B. 1922 - Les argonautes du Pacifique occidental, trad. fr. 1963. Paris, Gallimard, 606 p.

Macfadyen G., Salz P. \& Cappell R. 2011 - Caractéristiques de la pêche artisanale en Europe,

Département thématique des politiques structurelles et de cohésion, Pêche. Bruxelles, Parlement européen, $174 \mathrm{p}$.

Ministère des finances, Bulletin officiel des impôts, $n^{\circ}$ 43, 4 mars 2005 (Consulté le 28 mai 2015 http// : www11.minefi.gouv.fr/boi/boi2005/5fppub/textes/5b1305/5b1305.htm).

Règlement (UE) 508/2014 relatif au fonds européen pour les affaires maritimes et la pêche.

Règlement (UE) 1380/2013 du parlement européen et du conseil du 11 décembre 2013 relatif à la politique commune de la pêche.

Règlement (CE) 1198/2006 du conseil du 27 juillet 2006 relatif au fonds européen pour la pêche. Règlement (CE) 26/2004 de la commission du 30 décembre 2003 relatif au fichier de la flotte de pêche communautaire - annexe $\mathrm{i}$ - tableau 3 sur la codification des engins de pêche. 
Règlement (CE) 2371/2002 du conseil du 20 décembre 2002 relatif à la conservation et à l'exploitation durable des ressources halieutiques dans le cadre de la Politique commune de la pêche.

Règlement (CEE) 3760/ 92 du conseil du 20 décembre 1992 instituant un régime communautaire de la pêche et de l'aquaculture.

Règlement (CEE) 170/83 du conseil du 25 janvier 1983 instituant un régime communautaire de conservation et de gestion des ressources de pêche.

Reyes N. 2012 - Les pêcheurs aux petits métiers du littoral languedocien. Diversité des savoirs et des pratiques. Paris, mémoire de Master 2, EDTS-MNHN, 119 p.

Rieucau J. (Ed.) 1990 - Les gens de mer. Sète en Languedoc. Paris, L'Harmattan, 280 p.

Tempier E. 1985 - Mode de régulation de l'effort de pêche et le rôle des prud'homies. Thèse, Université d'Aix-Marseille II, Faculté des sciences économiques, 154 p.

\section{NOTES}

1. Ce pourcentage tendrait d'ailleurs à augmenter puisqu'il y a eu au cours des dernières années plusieurs destructions de chalutiers et de thoniers-senneurs.

2. Règlement (UE) 1380/2013 du Parlement européen et du Conseil du 11 décembre 2013 relatif à la politique commune de la pêche.

3. L'article L 931-2 du Code rural et de la pêche maritime définit « La société de pêche artisanale [comme étant] une société soumise au régime d'imposition des sociétés de personnes ou une société à responsabilité limitée et dont $100 \%$ des droits sociaux et des droits de vote sont détenus par un ou des pêcheurs qui en assurent en droit la direction et sont embarqués sur le ou les deux navires dont la société est totalement propriétaire ou copropriétaire majoritaire, ou qu'elle détient en copropriété avec un armement coopératif ou une société [...] agrée dans le cadre d'une accession progressive à la propriété dans un délai qui ne peut excéder dix ans ".

4. Décret 93 - 33 du 8 janvier 1993 relatif au permis de mise en exploitation des navires de pêche.

5. L'expression de "pêche côtière » est présente dans chacun des textes de la PCP sans qu'une définition n'y soit clairement établie. Elle est suggérée par les tableaux figurant en annexe qui délimitent la bande côtière à la distance des 12 milles marins. Règlement (CEE) 170/83 du conseil du 25 janvier 1983 instituant un régime communautaire de conservation et de gestion des ressources de pêche; Règlement (CEE) 3760/ 92 du Conseil du 20 décembre 1992 instituant un régime communautaire de la pêche et de l'aquaculture; Règlement (CE) 2371/2002 du Conseil du 20 décembre 2002 relatif à la conservation et à l'exploitation durable des ressources halieutiques dans le cadre de la Politique commune de la pêche ; Règlement (UE) 1380/2013 du Parlement européen et du Conseil du 11 décembre 2013 relatif à la politique commune de la pêche.

6. La limite des 12 milles marins est calculée à partir de la ligne de base ; 1 mille marin équivaut à 1,8 kilomètres.

7. Document du Ministère des finances, Bulletin officiel des impôts, $n^{\circ} 43,4$ mars 2005. En effet, certains navires aux « petits métiers » s'éloignent jusqu'à 20 milles des côtes. C'est notamment le cas des «petits métiers » qui disposent d'une Autorisation européenne de pêche pour la capture $\mathrm{du}$ thon rouge.

8. Cf. Le Règlement (CE) 508/2014 relatif au Fonds européen pour les affaires maritimes et la pêche et le Règlement (CE) 1198/2006 relatif au Fond européen pour la pêche. 
9. La liste des engins remorqués qui entrent en conflit avec la définition de "petite pêche côtière » figure dans l'annexe I du Règlement (CE) 26/2004 de la Commission du 30 décembre 2003 relatif au fichier de la flotte de pêche communautaire.

10. Décret 93-1342 du 28 décembre 1993 relatif aux conditions d'exercice des fonctions de capitaine et d'officier à bord des navires de commerce, de pêche et de plaisance.

11. Travail de terrain réalisé par $\mathrm{N}$. Reyes.

12. Les espèces cibles sont les espèces faisant l'objet d'une exploitation volontaire par les pêcheurs.

13. Cela concerne notamment les anguilles (Anguilla anguilla) et les daurades royales (Sparus aurata) qui sont des espèces très recherchées par les pêcheurs professionnels.

14. Le lido est un terme utilisé pour désigner le cordon littoral qui ferme une lagune.

15. Les joutes nautiques sont un sport régional qui consiste à la confrontation de deux jouteurs montés sur des barques. Le but étant d'envoyer son adversaire à l'eau au moyen d'une lance.

16. Quelques embarcations disposent tout de même d'une roue hydraulique permettant de faire remonter les filets.

17. Dans le Règlement (UE) 1380/2013, l'effort de pêche est, pour un navire de pêche, le produit de sa capacité et de son activité ; pour un groupe de navires de pêche, la somme de l'effort de pêche de l'ensemble des navires du groupe.

18. Le gangui, qui désigne à Sète un filet fixe, a changé de sens et désignait en Méditerranée orientale une forme de chalut à ailes (Gourret 1934 [1894], Dupouy 1955). C'est la forme en V terminée par une nasse en poche qui rapproche les deux types de filets, fixes ou traînés.

19. Pour plus d'informations au sujet de ces institutions se référer notamment aux écrits de Féral $(1980,1987)$; Frangoudes (1995) et Tempier (1985).

20. «Une définition utile et opérationnelle des petites pêches côtières doit au moins inclure la taille du navire, la polyvalence des engins, la mesure dans laquelle l'engin est actif ou passif et le niveau de dépendance vis-à-vis des eaux territoriales nationales » (Ifremer $2007: 30$ ).

\section{RÉSUMÉS}

À partir d'une double approche en anthropologie juridique et en ethnoécologie, cet article analyse la catégorie des pêches aux "petits métiers ». Ce segment présente plus de $80 \%$ de la flotte de pêche en Méditerranée française. Cependant, en dépit de son importance on relève une réelle imprécision dans la définition des catégories professionnelles pratiquant cette activité halieutique. Plusieurs expressions existent pour désigner les pêches aux "petits métiers » on parle alors de "pêche artisanale ", " petite pêche ", "pêche côtière " ou encore "petite pêche côtière ». Nous exposerons d'abord la terminologie présente dans les textes juridiques européens et français pour parler de ces pêches aux «petits métiers ».

Pour comprendre les limites de chacune de ces expressions, nous décrirons ensuite les techniques en concentrant nos observations sur les activités de pêche languedocienne exercée dans les lagunes. En effet, les techniques sont le moyen par lesquels le pêcheur accède à la ressource halieutique (poissons, mollusques et crustacés). Nous chercherons ici à dépasser la seule dimension technique afin de prendre en compte la manière dont le pêcheur les met en pratique. Nous montrons ainsi l'étendue de la diversité des pratiques existantes, comme celle des savoirs, des perceptions et des manières de travailler. 
Il apparaît donc que c'est cette diversité des techniques, employées dans divers écosystèmes aquatiques, qui constitue l'élément principal distinguant la pratique des pêcheurs aux "petits métiers » des autres pratiques de pêche. Une dénomination unique pour désigner l'activité de pêches aux "petits-métiers " donne l'illusion d'une catégorie homogène, là où en réalité sont réunies des pratiques de pêche extrêmement variées. C'est donc cette diversité qui constitue, pour les pêcheurs, la caractéristique fondamentale de cette activité. Compte tenu du décalage entre les définitions juridiques et les réalités du terrain, nous concluons cet article en proposant six paramètres qui permettent de caractériser les pêches aux «petits métiers " telles que considérées par les pêcheurs ».

The purpose of this article is to analyse through a double approach in legal anthropology and ethnoecology the meaning of « small trade » fishing. This segment presents more than $80 \%$ of the fishing fleet in the French Mediterranean sea. However, despite its importance, the definition of the professional categories practising this marine activity is still imprecise. Several terms, which are not synonymous, designate non industrial fishing : artisanal, small scale, coastal, small scale coastal fishing. We will first present the terminology used in European and French legal texts. Then, to understand the limits of each of these expressions, we will focus on the fishing activities in lagoons of the Languedoc Region. Indeed, the techniques are the mean through which a fisherman accesses marine resources (fish, mollusks, shellfish). We will try to go beyond the sole technical dimension to take into the use of each of these techniques. Thus, we will show the scope of the diversity of existing practices, as well as the knowledge, perception and ways of working.

It appears that the diversity of the techniques used in different marine ecosystems is the main element that differentiates small trade fishermen from other fishermen. A single denomination for artisanal fishing gives the illusion of a homogenous category where extremely various fishing practices do exist. It is this diversity that constitutes, for the fishermen, the core characteristic of their activity.

Considering the gap between the legal definitions and the realities of the field, we conclude this article by proposing six parameters which allow to characterize fishing in the "small trade " business such as considered by the fishermen.

\section{INDEX}

Mots-clés : pêche, Méditerranée, ethnoécologie, droit, techniques artisanales, petits métiers, lagune, diversité des pratiques, règlementation européenne

Keywords : fishing, Mediterranean, ethnoecology, law, artisanal techniques, small trade, lagoon, diversity of practices, European regulations

\section{AUTEURS}

\section{NASTASSIA REYES}

Muséum national d'histoire naturelle - Musée de l'Homme - UMR 7206 Éco-anthropologie et Ethnobiologie - 17 place du Trocadéro 75016 Paris

Institut de Recherche pour le Développement - UMR MARine Biodiversity, Exploitation and Conservation (MARBEC) - Avenue Jean Monnet - CS 30171 - 34203 Sète

nreyes@mnhn.fr 


\section{SERGE BAHUCHET}

Muséum national d'histoire naturelle - Musée de l'Homme - UMR 7206 Éco-anthropologie et Ethnobiologie - 17 place du Trocadéro 75016 Paris

bahuchet@mnhn.fr

\section{JEAN-DOMINIQUE WAHICHE}

Muséum national d'histoire naturelle - Musée de l'Homme - UMR 7206 Éco-anthropologie et Ethnobiologie - 17 place du Trocadéro 75016 Paris

wahiche@mnhn.fr 